\title{
Evaluating the Pleasures of Agency in Shiva's Rangoli, a Tangible Storytelling Installation
}

\author{
Saumya Gupta \\ University of California, Irvine \\ Irvine CA, USA \\ saumya@uci.edu
}

\author{
Theresa Jean Tanenbaum \\ University of California, Irvine \\ Irvine CA, USA \\ ttanen@uci.edu
}

\begin{abstract}
The experience of agency has been centralized in interactive digital storytelling research. Current approaches to agency place the actions of a reader into direct conflict with the authorial intentions of an interactive storyteller by privileging the authorial pleasures that come by interacting with the story's plot. In this paper we present the design and results of an empirical study of Shiva's Rangoli, a tangible interactive narrative system that supports meaningful interactions with the narratives that are decoupled from character actions and plot outcomes. Shiva's Rangoli allows readers to change the emotional, presentational, and aesthetic context of the story by giving them control over the ambience of the installation space where it is experienced, through a tangible interface. From our study, we identify three interaction styles or strategies undertaken by readers that reframe readers as Story Supporters, Meaning Makers, and Story Controllers to explore the pleasures of bounded agency within interactive storytelling.
\end{abstract}

\section{Author Keywords}

Agency; Interactive Storytelling; Interaction Styles; Diegetic Interface; Narrative; Tangible Interface; Ambience

\section{CSS Concepts}

Human-centered computing Human Computer Interaction

\section{INTRODUCTION}

Design and research into interactive digital storytelling (IDS) and interactive narratives has long concerned itself with questions of the authorial role of the player or reader. Magerko and Laird argued that one of the central challenges of interactive storytelling was to balance the desires of the interactor to act freely against the desires of the designer to create a satisfying plot [19]. Thue et al. argue that interactive storytelling is fundamentally about deferring the act of authorship until the player can arrive and provide essential missing information in the moment of play [46]. These

Permission to make digital or hard copies of all or part of this work for personal or classroom use is granted without fee provided that copies are not made or distributed for profit or commercial advantage and that copies bear this notice and the full citation on the first page. Copyrights for components of this work owned by others than ACM must be honored. Abstracting with credit is permitted. To copy otherwise, or republish, to post on servers or to redistribute to lists, requires prior specific permission and/or a fee. Request permissions from Permissions@acm.org.

DIS '19, June 23-28, 2019, San Diego, CA, USA

(C) 2019 Association for Computing Machinery.

ACM ISBN 978-1-4503-5850-7/19/06 ..\$15.00

https://doi.org/10.1145/3322276.3322380 approaches to interactive storytelling all emphasize the pleasures of unrestricted agency, often taken to mean the power to act freely within a game or simulation $[2,13]$. The end-state for systems that support this form of player-asauthor agency is often some sort of sophisticated engine for simulating social situations, such as Comme il Faut [22] or a massive unwieldy storyworld builder, like Crawford's now (possibly, temporarily) defunct Storytron [10] These systems facilitate player action often at the expense of a well-crafted story. There is no question that one of the pleasures of experiencing digital media is "the satisfying power to take meaningful actions and see the results of our decisions and choices" as Janet Murray defined agency in 1997 [23], but research and development around narrative games and IDS has elevated this pleasure to the point of disregarding other possible enjoyable user experiences. Consequentially, there are comparatively few studies of how players engage with an interactive story when the design does not actively encourage or support interactions at the level of the plot events and character actions. There are many possible approaches to this design space: in this paper we discuss one possible direction for new forms of interactive storytelling.

The research community has voiced the importance of finding a balance between the reader's interaction and the author's story plot $[20,23,44]$. As mentioned above, many researchers have advocated for giving readers authorial control over the story's progression. Such plot-centric approaches generally allow the reader to choose between different outcomes or 'branches', often favoring the reader's interactions over an impactful story [38]. It puts the onus on the author to write multiple branches, where they may have to deal with combinatory explosions. It can also cause anxiety and stress for a reader because the cognitive load of the "path-not-taken" accumulates over the experience [1]. We believe that dissociating the reader's interactions from the story's plot can alleviate these problems, while also exploring different forms of readerly pleasure that come from non-authorial interactions.

We have created a new tangible interactive narrative installation called Shiva's Rangoli. Unlike many research systems in IDS, Shiva's Rangoli is designed to decouple player interaction from specific plot events and character actions. Instead, interactors with the system are given the power to compose the ambience of the space in which the narrative is experienced, subsequently setting the emotional tone of the story and the ambience of their space. They 
interact with the system by manipulating the lighting of the space where it is experienced, the music that is playing in the background, the ambient soundscape of the experience, and (indirectly, via their other choices) an ambient video [4] that plays on a screen in the space. Readers of Shiva's Rangoli interact with the system and ambience through a tangible diegetic interface [7] that exists physically in the world of the reader and conceptually within the fictional world. We intended this narrativized interface [5] to act as a bridge between the reader and the story, so that readers feel as if they are a part of the story world. The Tangible User Interface (TUI) [16] at the center of this interaction is modeled after the Indian tradition of Rangoli making - a ritual and artistic practice where patterns are drawn with colored sand, flower petals, or other materials. The sum of these various elements is an almost ritualistic experience of a narrated story within a physically and sensorially immersive environment where the mood and tone of the story and the space responds to the preferences of the reader. Discussions regarding the diegetic nature of this piece have been elaborated in our previous work [14]. This work does not focus on the cultural aspect of the system as it will be addressed in future work.

In this paper we present the design of Shiva's Rangoli, along with one subset of data from an extensive interview and observational study of readers interacting with the installation. While our interviews uncovered many themes such as interactions with diegetic interfaces, interactive ambiences, taking on a character's role, and cultural aspects of the system; we focus our findings here on how interactors articulated their experience of agency within the system. Although our participants were only able to change the ambient, emotional, and aesthetic elements of the experience, we still identified three "interaction styles" that reflect different agentic pleasures. These include:

- Story Supporters who took pleasure in creating aesthetic contexts that reflected their understanding of the narrative they heard.

- Meaning Makers who expressed a feeling of agency over the emotional tone of the story but not the plot

- Story Controllers who experienced an illusion of control over the plot of the story at key points.

These three different levels of agency represent forms of narrative pleasure that have not been addressed by current IDS systems. While many of these pleasures may exist in contemporary story-based experiences, we believe that many designers do not set out to elicit these forms of agency. Identifying these interaction styles is this work's contribution to both the design and analysis of narrative experiences in interactive storytelling and games. We argue here that decoupling reader interactions from the specific formal narrative events of an interactive story doesn't eliminate the pleasures of agency, because agentic pleasure is a richer and more diverse experience than has been traditionally recognized within game studies and IDS. We close the paper with a discussion of the design lessons of the three interaction styles that we have identified. We clarify that through these interaction styles, our intent is not to claim any generalizability from our results but instead to identify actual strategies undertaken by interactors with a novel system that can guide designers and theorists of IDS. Although we have referred to previous work in narrative based games, Shiva's Rangoli is not a game; it is an interactive storytelling experience. For specifying the 'interactor' of the system, we have used the term 'player' when we talk about previous work in narrative based games, and 'reader' in reference to interactive storytelling experiences, including Shiva's Rangoli.

\section{BACKGROUND}

\section{Agency in Games and Interactive Storytelling}

In 1997 Janet Murray's Hamlet on the Holodeck formulated agency in terms of making meaningful choices [23] in IDS, which was further enshrined in the game design discourse by Salen and Zimmerman in 2004's Rules of Play [30]. The concept of meaningful choice has often meant giving players the ability to decide on the outcomes of a sequence of narrative events structured into a plot $[8,25,26,37,46]$. While many works acknowledge the need to find a balance between the reader's interactions and the author's plot $[20,23,44]$ this framing of the problem places the desires of the player into conflict with the storytelling goals of the designer [19]. We argue that this conflict need not take place.

We turn to recent reformulations of agency to consider alternative approaches to this core pleasure. Roberts et al. have successfully demonstrated that players need not have control over the outcome of an interactive story in order to feel the pleasures of self-determination within one [27]. Their work shows how players may be manipulated into taking actions intended by the designer through the use of social influence techniques from social psychology. Influenced participants have little real control over the outcome of events, but they report experiencing the pleasures of agency and efficacy, nonetheless. Similarly, Tanenbaum and Tanenbaum consider how the pleasures of taking action within games can be experienced as agentic, even when those actions are pre-determined, or decoupled from the act of choosing an outcome [44]. They propose an approach to agency that emphasizes "commitment to meaning" whereby the actions of the player align with the narrative intentions of the designer to produce the pleasures of meaningful action without relying upon player choices to determine narrative outcomes. Iten et al. conducted a study to identify choices that are meaningful in narrative based games [17]. One of their findings supports the claims above, suggesting that the impact of what one has done and felt, and the ability to choose how to perform a task is more important than the outcome of the task itself.

Informed by these perspectives, we designed Shiva's Rangoli to support interactions that mirror and reinforce the actions 


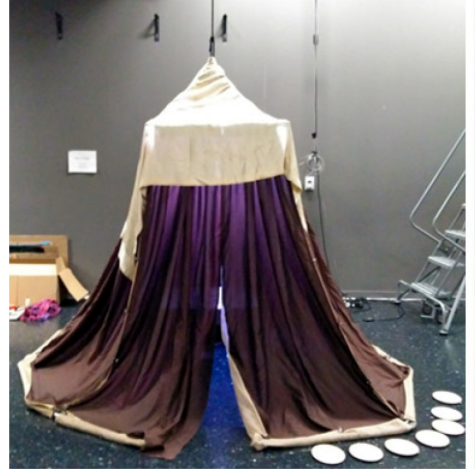

Figure 1: a) The installation space

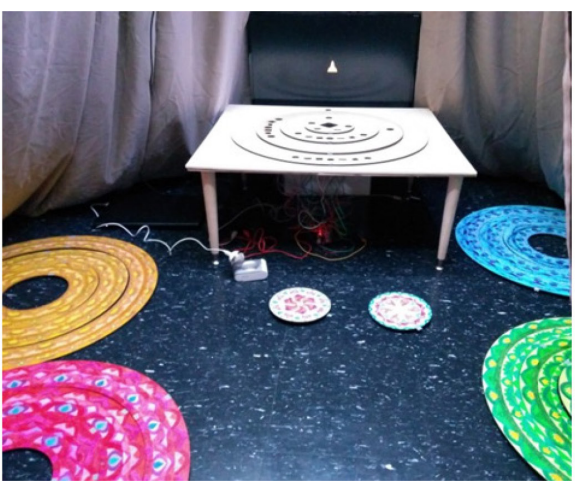

b) inside the tent

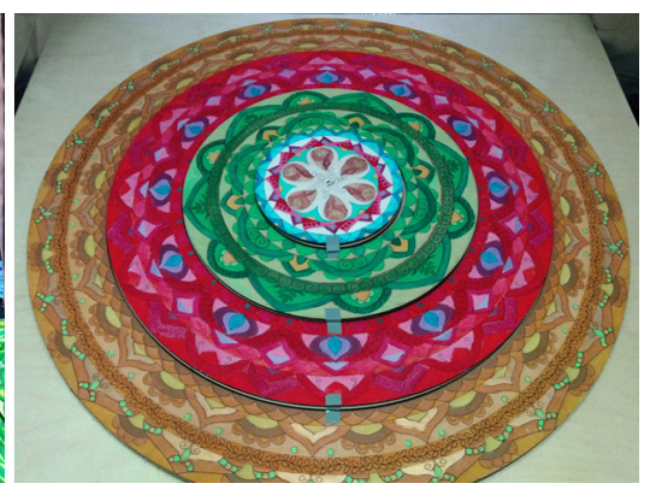

c) the interface of the main character. This design allows us to explore the pleasures of agency in a context where reader actions are decoupled from interactive narrative's plot.

\section{Non-Plot-Centric Interactive Storytelling}

While most research into interactive storytelling focuses on providing players with the ability to alter plot outcomes, there have been a few interesting exceptions. In the IDS community, Seif El-Nasr's system, Mirage, changed the visual language of the story to reflect different models of user preferences [32-34], responding to player interactions outside the narrative's plot. The most direct inspiration for Shiva's Rangoli is an interactive storybook called Scarlet Skellern and the Absent Urchins (SSAU) [42,43]. Scarlett Skellern is a digital storybook that captures the affective preferences of the reader and enables them to interact with the story's mood by changing the instrumentation of the score, the ambient soundscape, and the color palette of the story [42]. Inspired by this project, Shiva's Rangoli gives readers the ability to change the affective tone of the narrative by shaping the ambience of their physical space. In game studies, Nay and Zagal discuss how 'inconsequential choices' that deliver the same outcome irrespective of the choice can help players reflect on the motive of their actions, shifting the focus to reflection on morals rather than what outcome to choose [24]. They state that the player should be able to contribute to the crafting of the protagonist's perspective, allowing them to impact the moral character without changing the plot. In all these examples, players are actively engaged with a system that is generating rich audiovisual feedback in response to their actions, but they are unable to impact the events that play-out in the story.

\section{Tangible Interactive Storytelling}

Tangible interfaces are a part of the reader's physical environment as opposed to being limited to computer screens [16]. They can create immersive experiences for interactive storytelling by connecting readers to the story $[15,20,21,41,45]$. The interface design of Shiva's Rangoli has been influenced by literature that emphasizes how interfaces can make readers feels as if they are a part of the story world, shifting the focus from authorial pleasure to meaningful participation $[3,5,40]$. Bizzocchi et. al describe 'narrativized interfaces' as an approach to integrate the narrative with the design of the interface in story-based systems, enabling players to perform the role of the embodied character [5]. Bordwell and Thompson define the term 'diegetic' as "things that exist within the world of the film's narrative."[7]. Harley et. al state that "diegetic tangibles present a compelling alternative to tangibles as metaphorical objects or as input devices" [15] and cite The Reading Glove [41,45] as an exemplar of this kind of design. They state how these kinds of interfaces can connect the reader's world with the story world. In Shiva's Rangoli the TUI and the configurable environment both act as diegetic narrativized interfaces, as they simultaneously exist in the reader's physical world and the story's fictional world. Harley et al. recently proposed a framework to classify 21 tangible storytelling systems [15]. They argue that very few works make the user a central part of the story, often instead positioning them as an external observer and explorer, without providing them a sense of responsibility or ability to have an impact on the story. With Shiva's Rangoli, we investigate ways to make readers feel as if they are a part of the story world, have a stake in how the narrative unfolds, while dissociating impact with the story's plot.

\section{Interactive Ambient Environments}

The works below inspired us to use ambient settings as an interactive medium in Shiva's Rangoli. Ross and Keyson designed a tangible expressive interface to sculpt the ambiences in their living room by controlling lighting, audio, and video art [28]. Li and Jianting propose an intelligent space that portrays a person's emotions based on their movement, using ambient and audio visuals [18]. In the LIT ROOM, storytellers create expressive ambient environments that are evocative of the picture books being read to children [31]. Wassermann et al. [47] used different ambient presentational styles to express emotions and found that participants were able to associate emotions to ambient settings, which is why we used interactive ambient environments for setting an emotional context.

\section{DESIGNING SHIVA'S RANGOLI}

The design process of Shiva's Rangoli was iterative. We collaboratively worked on the interaction design and created cardboard prototypes to test with our colleagues and make improvements. Shiva's Rangoli enables readers to craft the 
ambience of their space through a culturally inspired narrativized tangible interface [5] subsequently setting the mood and tone for the narratives, as they hear two interleaved stories.

\section{The Interface}

Inspired by Ranjit Makkuni's work on cultural interfaces [48] we designed the TUI to mimic the Indian art form of Rangoli making. Rangolis are colorful patterns that are made on floors with colored sand, flower petals, or other materials, during various festivities. The readers assemble Rangolis in the installation space by placing different concentric rings together. The interactive space is enclosed by a pavilion and inside the tent lies a table, twelve artistically patterned rings and two center pieces, as shown in Figure 1a and 1b. There are four sets of three rings each, where each set represents a particular mood. The yellow rings represent happy, green is calm, blue represents sad, and red is angry. This design choice was based on the color to emotion mapping proposed in the eMoto project [12,35] and is grounded in Russell's circumplex model of affect [29]. Any three of these twelve rings along with a center piece, can be placed on the table in a concentric way to create a Rangoli as shown in Figure 1c. The rings set different ambient effects based on the mood that they represent. The outermost ring set the lighting effects of the space, the middle ring sets sound effects and the innermost ring sets the music. The two center pieces determine which of the two narratives is played back. The tabletop is a foot-tall, affording readers to sit down on the floor while assembling the Rangolis, imitating the traditional style of creating Rangolis on the floor.

\section{Ambient Effects}

The rings are placed on the table to set the ambience inside the tent according to the associated moods. The yellowhappy rings set a warm, upbeat, and chirpy atmosphere. The green-calm rings create a soothing and soft environment. The blue-sad rings create a low and dull ambience. The red-angry rings create a stormy, thundering environment. Once the reader assembles a Rangoli, an ambient video is displayed which reflects the aggregate mood created by the reader. The ambient effects are based on nature and river visuals and sounds, in coherence with the theme of the narratives (see next section for more details). These effects were decided through a series of iterative user tests. We do not make any claims on the universality of these mappings with emotions. The media content (sound, music, and video) used belongs to artists who have listed their work under the creative commons license agreement of YouTube. Figure 2 shows the different ambient spaces created when all rings of the same colors are placed. Readers can place any combination of the 12 rings available, setting ambient effects belonging to different moods. Once the reader completes assembing an entire Rangoli, a chapter from the story represented by the center piece is narrated. The system calculates the overall mood value represented by the Rangoli, and the chapter adapts to the emotional tone set by the reader. Mood-based textual elements in the narrative change accordingly (see next section for more details). After the narration is complete, readers may change parts of the Rangoli before starting the next chapter. Each story consists of 10 chapters that are played in a linear way.
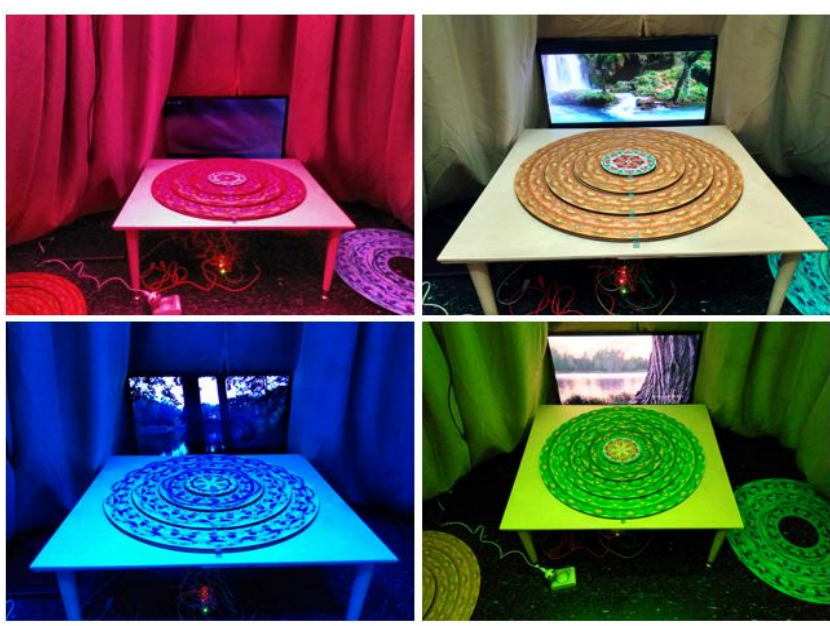

Figure 2. Ambient Effects

\section{Narratives and Agency}

The two stories, called 'the river' and 'the medicine', have been written by the first author and are inspired by Indian mythology, with a magical river being a central theme between both stories. The stories highlight the importance of the cycle of creation, maintenance, and destruction. In the narratives, the protagonist Shiva is a powerful God who makes Rangolis to express his emotions and decisions. The God's emotions trickle down into the human world and the Earth's environment reflects Shiva's mood and the colors of his Rangolis. For example, when Shiva is angry, the fictional world's skies become dark and bloody with heavy rains and raging thunderstorms. Readers are invited to assume the role of Shiva and create these Rangolis as a reflection of Shiva's emotions. In this way, readers are given agency over Shiva's mood as well as the ambience of the space in the tent and the fictional world. Readers are endowed with responsibility for Shiva's emotions and how he impacts the story world [40]. Furthermore, the act of creating a Rangoli enables readers to enact Shiva's actions, aiming to aid transformation [40]. Both the Rangoli and the dynamic ambiences serve as diegetic bridges, as they are a part of the story world and real world [5,7], acting as boundary objects [36]. The ambient effects are inspired by water, to give readers a sense of being near the river.

Each chapter has two versions: an emotionally positive version and an emotionally negative version. For simplicity, we take only the valence of the mood created by the reader into consideration, grouping happy and calm as positive and sad and angry as negative. After the reader has assembled the Rangoli for a chapter, the system calculates the overall mood / valence value of the Rangoli rings assembled by the reader and triggers one of the two versions. In both versions, the plot remains the same, but the emotional context of the story 
changes. Shiva's mood, the river's waters, the weather in the fictional world, and some minor consequences not impacting the plot, are different between both versions of each chapter. Since the plots of the stories do not change, the narratives do not branch out at any point. In addition to the changes in text, the narrator's tone of voice changes to augment the emotional tone set by the reader. In this way, readers have agency over Shiva's emotions and attitude, changing the way they portray Shiva. Here are the emotionally positive and negative versions from one of the chapters:

Positive version: "Brahma and Vishnu approached Shiva and as they looked upon the vibrant Rangoli filled with shades of yellow and green, they were delighted to see that Shiva was in one of his good moods. The skies were light, harmonious and chirpy reflecting Shiva's pleasure. Brahma and Vishnu never thought twice before approaching him on such days, and so they decided that it was the perfect time to confront him on one of their never-ending arguments, Shiva's destruction".

Negative version: "Brahma and Vishnu approached Shiva, and as they looked upon the bold Rangoli filled with shades of blue and red, they understood that Shiva was not in the best of his moods. The skies were dark, gloomy, and enraged, reflecting Shiva's displeasure. Brahma and Vishnu always thought twice before approaching him on such days, yet today they mustered the courage to confront him on one of their never-ending arguments, Shiva's destruction."

As described above, Shiva's mood and the state of the fictional world change but the consequence of Brahma and Vishnu confronting Shiva over his destruction does not change. In both stories, Shiva's Rangolis are acknowledged frequently to help readers learn how their choices and actions map into the story world, and how the colors map to the moods. This design gives readers opportunities to act within bounded agency that enables them to interact with the mood and aesthetics of the story, enact Shiva's role, accept responsibility for the characters, and shape their ambient space that directly impacts the narrative's tone. Since the story plot does not change, readers can experience the pleasures of the above interactions, without being burdened by possible story branches.

\section{Design Iterations}

Shiva's Rangoli went through multiple interaction design and hardware iterations over a period of ten months. Our aim was to explore non-authorial interactive possibilities. Inspired by previous work [42], we decided to explore the theme of aesthetics and mood in the narrative through ambient effects. We first decided on the Rangoli interface and how its rings would control the ambience of the space. In the first design iteration, we connected different mood settings to different stories, making one story trigger for a positive affect ambience and the other story trigger for the negative affect ambience. This design was paper prototyped and tested in 'wizard-of-oz' style with four of our colleagues who had no knowledge about this project. We quickly realized that this design felt more like solving a puzzle rather than affording engagement with the narrative. At this point, we came across Harley et. al's framework [15], that encouraged the use of diegetic objects to connect readers with the narrative and called out the need for experiences that positioned readers inside the story world, rather than making them feel as if they are solving a puzzle. We decided to make both the Rangoli interface and the ambient effects diegetic. This interaction design was tested with cardboard prototypes with four more colleagues. We learned that Shiva's actions had to be acknowledged multiple times in the narrative to help readers understand the diegetic nature of the interface. We also added more emotion based contextual cues in the narrative such as 'stormy nights', or 'blissful lands.' We explain our process of story writing to accommodate affect based changes (without changing the plot) in the design lessons section. After these iterations, we were confident with our interaction design and proceeded to make the hardware. Before running the study, we conducted three pilot tests. We observed that people needed some cultural context about what Rangolis are and who Shiva was before the experience started. We also observed that in the first few minutes of the experience, people were overwhelmed by listening to the narrative as well as exploring ambient effects. To reduce the novelty effect of the system, we added a demo to help people explore the ambient effects and get acquainted with the system's working before the narratives begun to play.

\section{STUDY}

This paper presents a subset of the data collected in our study of Shiva's Rangoli related to how readers interacted when they had agency over the emotional and aesthetic context of the story. We also studied how diegetic interfaces and ambient settings facilitated this interaction by connecting readers to the story world, which has been elaborated in our previous work [14]. We recruited participants through postings on Facebook, flyers on UC Irvine campus, and through word of mouth from the faculty, alumni, and student population at UC Irvine. There were no limitations on participation except for the ability to comprehend English. Ages of participants ranged from 18 to 54 with the majority being below 34.9 participants identified as females and 14 as males. They belonged to different nationalities in which nine identified as Americans, seven as Indians, four as Asians and three as other. Majority of the participants came from information and computer science disciplines and a few belonged to social sciences. Most Indian participants had practiced the art of Rangoli making before and were acquainted to other Indian mythological folk tales. Participants from other nationalities did not know what Rangolis were, and only few had heard of Shiva before. We conducted 23 study sessions in our lab that lasted for a duration of two hours each. According to the study protocol, participants were first introduced to the concept of Rangolis 
by showing them pictures of people creating traditional Rangolis, and by telling them how and when Rangolis are made in India. They were told that Shiva was known as the God of destruction in Indian mythology. They were informed that the Rangolis they created in the tent would be created by the protagonist Shiva as well. They were not given any information about the impact their choices had over the narratives. They were given an overview of basic interactions with the system, followed by a practice session which allowed them to explore all the ambient effects without hearing the narratives. The stories began to play after the participants signaled that they were ready. The experience was 30-45 minutes long and participants were asked to think aloud while interacting with the system. The researcher sat outside the tent and took notes. The interaction was followed by a semi-structured interview where we asked them about their understanding of the story, their mental model of the system, thoughts on the ambience and moods, their decision-making process, and their perceived role in the experience. We analyzed the interview transcripts and the think aloud notes taken during the session starting with open coding and then moving into axial coding [9,11]. We identified and categorized various patterns from the data and constructed broader themes. We visualized these codes and themes with sticky-notes on a whiteboard and made meaningful connections.

\section{FINDINGS}

In our analysis of the data we identified three Interaction Styles that people adapted which were linked to their perception of agency over the narrative. We term these as Story Supporters, Meaning Makers, and Story Controllers. Story Supporters created ambiences to support their understanding of the narratives, without impacting the stories. Story Controllers reported a sense of some amount of control over the plot of the story especially when their actions were acknowledged in the narrative. Meaning Makers felt they had agency over the mood and tone of the story and the characters but not the plot. There were eleven readers who interacted as Meaning Makers, ten as Story Supporters and seven interacted as Story Controllers. These styles were not mutually exclusive as readers took on different interaction styles as they learned how the system worked. For each interaction style, we elaborate behavioral patterns, the motivations, and consequences of adopting the interaction style. We clarify that we are not generalizing reader behavior as people's interactions are too varied and complex to generalize, irrespective of the sample size. Rather, we observe how readers arrive at different strategies for making personal meaning within a context of restricted agency. This can shed light on practices of meaning making that have not yet been articulated within the IDS community.

\section{Interaction Styles}

Story Supporters: predicting what happens next

Behavior: Readers acted as 'Story Supporters' when they felt that they could not impact the story's plot, or the narrative's tone, believing that the stories were completely pre-written.
They found pleasure by creating ambiences and mood settings that matched the story's perceivably pre-scripted plot by predicting what would happen in the next chapter. For example, if they predicted chaos and destruction, they would use the red (angry) rings to create a supportive ambience. One reader re-created the environment as told in story (night / day) to put themselves in the Shiva's shoes. One reader believed that Shiva being the God of destruction was best represented by red (angry) rings and would create an angry ambience to support their perception of the character's personality.

P02: "It's almost like a play where I am the setting or
the backdrops or stage hand. You just foretold that
there is going to be an argument. Now I need to change
the mood because I need to support the story since that
is where the story is going."

P06: "I was more engaged in trying to match it (ambience) to the story and trying to predict what was going to happen, so I could put the right rings down for the environment. I kind of already figured that the stories were already there, so I just tried to play along with it."

Motivations: Readers said they were motivated to match the ambience to the story's plot as it immersed them in the experience and any deliberate defiance would break this engagement and cause disruption. Furthermore, they held themselves responsible for any mismatch between the ambience and the story's content. These readers believed they had no control over the story as they met a few occurrences where there was a mismatch between the ambience created and the story's plot (described in further sections), leading them to the conclusion that the story was completely pre-written. Some assumptions such as the story being pre-scripted due to the numerous branching possibilities, and the stories being well established mythological tales, led to this conclusion as well.

Consequences: These readers felt more immersed in the experience when the plot matched the mood setting, they created. They felt accomplished when the aesthetics they created matched the tone of the story.

\section{Story Controllers: Felt some degree of control over plot}

Behavior: These readers experienced control over the plot at certain points during the interaction, especially when the reader's Rangolis were acknowledged in the narrative. We term this as an 'illusion of agency' over the plot as the narrative did not branch out into a different story line at any point [44]. Readers felt that they controlled Shiva's actions and fate as they created ambiences to make him behave in certain ways. Some projected their own mood on the character by creating ambiences that matched their current mood.

P16: "Every time it (story) acknowledged me, I felt like I had accomplished something or changed the narrative." 
P19: "There were some places - for instance in the first story, the chapter ended with Shiva making a Rangoli, and Brahma and Vishnu would see the Rangoli to understand his mood. I think that's when I got a choice to make the Rangoli and that's how the story would progress."

Motivation: Readers felt in control of the story when the narrative acknowledged their Rangoli and specified the impact of the selected colors. They also felt a sense of responsibility towards the character and story outcome, which further guided this interaction style [40]. They felt more in control when the ambience matched the plot of the story, and when their choices had impactful consequences (even though the outcomes were the same). The narrative cue in the ending of the stories that acknowledged the Rangoli before its creation and cautioned readers of the consequences of their next choice particularly helped readers to feel in control.

\section{P21: "I felt like the reference to the Rangoli in the story prompted me to think more about it. And it went in the direction that I had created with the Rangoli, the happy ending in the second story and the uncertain ending in the first story were in line with my choices."}

Consequence: Two readers felt that they transformed into Shiva towards the end when the narrative acknowledged their Rangolis. One reader's goal was to end the narratives on a happy note which prompted her to create only emotionally positive ambiences, fearing that any negative ambience might end the story in a darker tone. This curbed the independence of exploration, changing the emotional tone, and making meaningful choices.

Meaning Makers: Agency of mood, tone, and perception Behavior: Readers expressed feeling that they could change the mood of the characters, the tone, and pace of the story through the ambience without changing the plot. The ambient and emotional setting helped them see the narrative through different perspectives and put them in certain moods which changed their feelings towards the story events. For example, one reader claimed that the ending of the story 'the river' seemed hopeful for a new beginning despite the destruction, as the ambience they created was calm and uplifting. One participant interacted with both the stories twice, and justified the endings very differently each time, based on Shiva's emotions and intent behind his actions, even though he acknowledged that the outcomes were the same. Readers used ambiences to enhance or diminish the feelings associated with the events of the story, however, the underlying emotion attached to a specific event depended on the story content and not the ambience. Readers created ambiences to support their prediction of Shiva's feeling, some placed themselves in his situation, and a few projected their own emotions on Shiva. At times, they also represented a mix of or varying intensities of different emotions through the Rangoli. Readers said they associated different meanings with ambiences over the course of the story in relation to the context of the plot. For example, one participant mentioned creating an angry ambience, but feeling relaxed about the same, as justice was being served in the story.

P13: "I felt because I had influenced the mood of the character, even though it didn't influence the progression, I felt like I was be able to tell my own story of his emotional ups and downs and that felt powerful to me. It is lot like being the director of the show because the director doesn't write the script. But the director sets the tone, ambience, what it looks and feels like."

P05: "I mostly went with the content of the story. In Shiva's story, just like Shiva I was annoyed with Brahma and Vishnu that they were complaining so much even though it was just me doing my job. I portrayed that with anger and sadness through the Rangoli."

Motivation: Participants reported that the interactive ambience encouraged this interaction style as it helped shape the reader's mood, evoke emotional responses, and interpret the narrative in different ways. Besides the ambience, the change in narrator's voice and tone helped set an emotional context. Participants also acknowledged that the story was written in a way that could be interpreted in different ways. Certain mismatches in the emotional context of the ambience and the plot helped readers conclude that they had no agency over the major events and narrative arc, and more agency over how the events unfolded. We observed that readers were committed to creating ambiences that would best represent Shiva's mood in a particular situation as they felt responsible for him.

\section{P05: "More than the story, emotion drives the story is my take on it. I find it very fascinating and necessary how emotional constructs and crafts shape how story is told, and not just in a content-based sense."}

Consequences: Readers felt they could personalize the story and just by inflexions in the mood and tone. They could interpret the narrative in various ways and had the freedom to represent Shiva in different shades depending on the emotions he expressed while carrying out his actions. A few felt that they transformed into Shiva, as there was no distinction between their own feelings, Shiva's emotions, and the narrative tone, enabling them to empathize with Shiva more. Table 1 summarizes the three interaction styles.

\section{Changing between Interaction styles}

Readers shifted between different interaction styles as they learnt how the system worked and the agency they had over the stories. During the first half, they were more exploratory, and they chose their favorite colors or ambient effects until they learnt how their choices impacted the narratives. They understood the working of the system with the help of contrasting ambient settings, a thoroughly explored demo, when the narrative acknowledged their Rangoli, and when the consequences of their actions were called out. 


\begin{tabular}{|l|l|l|}
\hline $\begin{array}{l}\text { Interaction } \\
\text { Style }\end{array}$ & Perceived agency & Behavior \\
\hline $\begin{array}{l}\text { Story } \\
\text { Supporters }\end{array}$ & $\begin{array}{l}\text { Created ambiences } \\
\text { to support their } \\
\text { understanding of } \\
\text { the narratives, } \\
\text { without impacting } \\
\text { the stories }\end{array}$ & $\begin{array}{l}\text { Predicted the story and } \\
\text { matched the story's } \\
\text { perceivably pre-written } \\
\text { plot }\end{array}$ \\
\hline $\begin{array}{l}\text { Story } \\
\text { Controllers }\end{array}$ & $\begin{array}{l}\text { Controlled the } \\
\text { story plot at key } \\
\text { points }\end{array}$ & $\begin{array}{l}\text { Felt they controlled the } \\
\text { story when the narrative } \\
\text { called out their actions } \\
\text { with the Rangoli }\end{array}$ \\
\hline $\begin{array}{l}\text { Meaning } \\
\text { Makers }\end{array}$ & $\begin{array}{l}\text { Felt agency over } \\
\text { the tone, pace, and } \\
\text { mood of the story } \\
\text { but not the plot }\end{array}$ & $\begin{array}{l}\text { Changed mood of story, } \\
\text { enhanced or diminished } \\
\text { feelings associated with } \\
\text { an event, saw different } \\
\text { shades of Shiva's } \\
\text { personality, and saw the } \\
\text { story from different } \\
\text { perspectives }\end{array}$ \\
\hline
\end{tabular}

Table 1: Summary of interaction styles

During the second half of the interaction, readers made more conscious decisions according to what they had learnt and the agency they felt over the stories. At times, the ambience and tone that readers created would not match the story's plot. Although each chapter was written to adapt to the mood created, a few chapters had essential plot elements that could not be represented in both happy and sad tones. In these cases, a happy ambience created during a sad chapter would result in a mismatch of emotional tones, breaking immersion. Similarly, an emotionally negative ambience would at times not match a relatively happier event, yet this case was not as noticeable as the former. Despite the negatives, the mismatches helped readers understand that they did not have complete agency over the plot of the narratives, the stories were pre-written to an extent, and there were no branching narratives. Participants acknowledged that the mismatches brought more attention and focus as it led them to question their understanding of the system. As people understood the agency they had over the narratives, their interaction style would change. For example, P02 started with the assumption of being able to control the plot, acting as a Story Controller. This assumption was removed due to the mismatch in the ambience and the plot at a few places, after which P02 switched between acting as Meaning Maker and Story Supporter depending on when Shiva was present in the story and when he was not respectively. Many readers felt like Story Controllers when their Rangolis had a significant impact on the story, especially towards the end. This shows the rich interactive possibilities that readers adapted throughout the experience, from exploration in the beginning to informed decision making later.

\section{Acting Meaningfully}

Story Supporter, Story Controllers, and Meaning Makers were motivated to create meaningful experiences for themselves. For this reason, they often changed the Rangoli rings and ambient settings after every chapter. Story Supporters said they wanted to create an engaging experience and avoided mismatches between the plot and the ambience as they did not want to break immersion. Furthermore, they held themselves responsible for any mismatches. Meaning Makers reported changing the ambience to put Shiva in certain moods, see various shades of his personality, and change the tone of the story. This enabled them to experience the narrative from different perspectives and personalize their experience. Story Controllers claimed that they created Rangolis to serve the narrative as they felt responsible for the fate of the characters. Readers were committed to creating ambiences that fit well with the narrative, rather than causing deliberate disruptions. One participant acknowledged how they went from choosing their favorite ambient effects and creating 'moods for themselves', to creating 'moods for Shiva', that would serve in his best interests. Readers acknowledged that creating ambiences for the narrative was more important than their choice of aesthetics for the Rangoli, their favorite colors, or what they wanted the ambience to be.

\section{P18: "My main goal was to hear a good happy story but that didn't come out until after I realized that my desire to have these characters have a good story was greater than my desire to troll around. Else I would have done all blue as that is my favorite color."}

This shows that the desire of the reader and the goal of the storytelling designer does not have to be in conflict with each other. These findings support Tanenbaum and Tanenbaum's claim of players being committed to experiencing the best version of the narrative rather than solely asserting dominance over the plot [44].

\section{Participant Reflections on Narratives}

Readers reflected on multiple aspects of the story as the experience was interactive and delivered a coherent narrative. Before the experience, many readers believed that destruction carried a negative connotation and creation carried a positive connotation. This led them to believe that Shiva, the God of destruction may be evil, a misconception that shifted as the experience progressed. After the experience, they realized how destruction was at times necessary to restore balance. One reader mentioned how change was possible only through the cycle of creation, maintenance, and destruction. One reader commented how free will in life may be an illusion and we may just be the puppets of the Gods. Another claimed that he became more aware of his own emotions and actions after the experience. We feel that the coherent and impactful plot of the narratives, the interactive and diegetic nature of the design, and the ability to experience the stories from Shiva's perspective all helped participants reflect on these story concepts. However, we had not anticipated this level of philosophical reflection.

\section{Cultural Experience}

Readers who were familiar with Indian culture and traditions reported that they found comfort in the experience. One participant mentioned how the experience brought her back home. They mentioned how this experience added to their 
knowledge and reinforced morals learnt from Indian mythology. Readers who were unfamiliar with Indian culture liked that they were able to experience the system through a different cultural perspective. A few found the story content interesting as they had not heard of different Gods having opinions and negotiating with each other, or Gods talking to humans as these concepts were different from what they had heard in their own cultures. They also compared the Rangolis to Mandalas and dream catchers. Readers were curious to know more about Indian mythology, culture, and Rangolis after the experience. They saw the installation as a fun and interactive way of being introduced to a new culture.

\section{DESIGN LESSONS}

The findings above show the diverse pleasures that readers experience within the context of bounded agency. We elaborate few design lessons that we learnt after our study. We highlight how certain aspects of the system enabled people to adopt the three interaction styles that we identified - Story Supporters, Meaning Makers, and Story Controllers, and how these styles could have been supported further. None of the three Interaction Styles mentioned above advocate for giving readers control over the plot. We do not ascribe any particular value judgement to these interaction styles, but merely represent possibilities that emerge when the reader's interactions are decoupled from the plot. We also elaborate the importance of leaving room for multiple interpretations for interactive experiences. We hope these design lessons can help researchers think about various forms of agency and ways to design for experiences with controlled agency.

\section{Leave room for interpretation within constraints}

We observed that 21 out of the 23 participants found a meaningful way to interact with the system by following one or more of the interaction styles. We believe this was possible because participants had enough room to understand the system and interact how they desired within the constraints of the experience. We conclude this was a successful design choice as the system did not demand rigid adherence to a specific expected reader behavior, but instead facilitated exploration. We find this approach leaves room for different interpretations and provides readers with an opportunity to create a reflective, meaningful and personalized experience for themselves (within the narrative framing provided). This also enabled readers to transition between different interaction styles. Despite being given the freedom to explore, readers were more interested in creating the best experience for themselves than in subverting the system and the intent of the designer [39].

\section{Designing for Different Interaction Styles}

\section{Designing for Story Supporters}

Story Supporters were motivated to interact in ways through which they could support a pre-written narrative, without asserting control over the plot of the story. In Shiva's Rangoli, readers tried to predict the next chapter of the story and match the ambience to their prediction. Future designs could guide the readers by hinting them of the upcoming events in the story, aiding them to follow the story through their interaction. Similar to Bizzocchi and Tanenbaum's recommendations, we observed that having well defined character personalities $[6,38,40]$ helped ground readers, as they predicted the character's actions based on his temperament. A few participants tried to re-create the environment of the story in our study, which implies that readers can be guided by describing the scene of the narrative or other elements over which they have agency. In Shiva's Rangoli, we gave readers agency over the mood and ambient setting. Other possible forms of interaction within bounded agency could be diegetic objects that traverse readers through non-linear narratives [41], or objects that can give different character perspectives of the story [21].

\section{Designing for Meaning Makers}

Meaning Makers personalized their experience within the bounded agency they were given, leaving control of the narrative arc to the author. In future designs, agency could be given over the character's personality [24], time lapses, or other elements that can help personalize their experience and derive different meanings. We observed that giving Meaning Makers enough room for making multiple interpretations of the story within controlled agency was important. It was useful to have contrasting effects of the selected form of agency, such as the varying effects in ambiences, as it facilitated readers to recognize how they were impacting the story. Meaning Makers did not have control over 'what' happened in the narrative, rather they decide 'how' the events of the story unfolded [40,44].

Based on our findings, we highlight lessons that we learnt while creating an experience where readers had agency over the emotional context of the story but not the plot. Writing the story first and then changing the narrative to adapt to different emotional tones, helped us deliver an impactful story without being apprehensive about integrating the reader's interactions. To give agency over the mood and tone of the character, we wrote the stories such that Shiva expressed contrasting intensities of various emotions through different dialogues that delivered the same underlying message. This way, readers were able to personalize their view of Shiva by experiencing different shades of his pre-written personality - A God trying to control his temper or a God with a bad temper. We realized that each event of a narrative could not be represented in various emotional tones. In these places, we did not compromise on the plot, rather we provided ways to enhance or diminish the emotion underlying an event by representing the situation in a better or worse way. Here is an excerpt of an intense scene from the 'river' where the Gods are concerned about the way humans are mistreating the river.

Better: "The Gods once again paid a visit to the humans in their dreams and asked them to restore the river's purity, reminding them of the old golden days that once existed." 
Worse: "The Gods once again paid a visit to the humans in their dreams and demanded them to restore the river's purity, warning them of the repercussions."

Both these dialogues express concern for the river, however the 'better' version tones down the tension in the scene whereas the 'worse' version adds to it. In such a scene, a happy ambience led to a break in immersion. However, the plot was not compromised to avoid these mismatches, as we saw how mismatches helped readers realize the agency they had over the narrative. We could have reduced these disparities by hinting the readers of the upcoming events in the next segment. In addition to the narrative, the interface played a critical role in the experience. The diegetic interface enabled readers to mimic Shiva's action of creating Rangolis, enabling them to connect with his role in the experience. We found that crafting diegetic interfaces in different ways and mapping the reader's action into the story world, facilitated them to enact a role and gave them some form of agency over the narrative. Additionally, giving readers a stake in the story and a sense of responsibility encouraged them to act meaningfully.

\section{Designing for Story Controllers}

Story Controllers felt in control of the story when the system acknowledged their actions in the narrative, mapped these actions to impact the story, and warned them of the repercussion of their choices. Since we did not want to change the story plot, the impacts of the reader's actions were inconsequential [24], that is the same outcome irrespective of the choice, as elaborated by Nay and Zagal. Giving readers a sense of responsibility in the narrative motivated them to think about their actions as many participants were concerned about the fate of the characters. We could have supported this interaction style further by guiding readers about what they had control over, so that they could make informed decisions without concentrating on solving the mystery of what they could impact. The diegetic interface also played a crucial role in this interaction style. Readers felt they had agency over the character's actions and could see the impacts of their choices play out because the reader's actions were mapped into the story world through the diegetic Rangoli interface. We found that warning readers of the consequences of their actions gave them a sense of control, irrespective of the fact that the choices were inconsequential. We do not imply that all diegetic interfaces will give an illusion of control, it is merely a means to achieve one of the many possibilities. We observed that a few readers who played as Story Controllers were very concerned over how the narrative would end, which curbed their freedom to explore and create a meaningful experience. We infer that providing agency as 'Story Controllers' while giving an opportunity for more insightful thinking and reflective possibilities is worth further research.

\section{CONCLUSION AND FUTURE WORK}

The goal of this work was to explore how readers interact with a storytelling system at the level of its emotional and aesthetic context, without impacting the plot of the narrative. We studied the interpretive possibilities, the pleasures of interaction when decoupled from choosing an outcome, and how readers interact within their perceived level of agency. Our findings reveal that readers felt three levels of agency over the narratives, which we call Interaction Styles: Story Supporters, Meaning Makers, and Story Controllers. A common behavior that we observed between these styles was that readers were committed to creating the best experience for themselves [44]. Story Supporters felt that the story was completely pre-scripted and created ambiences to support their understanding of the narrative, creating an immersive experience for themselves. Meaning Makers chose ambiences to best suit the needs of Shiva's emotional state and to see the narrative from different perspectives. Story Controllers felt they could control the story at key points and held themselves responsible for the outcomes of their decisions and the fate of the narrative. Based on these findings, we provide design lessons learnt for the three interaction styles identified. We also emphasize the importance of leaving room for accommodating multiple interpretations of the experience. We conclude that decoupling the reader's interactions from the plot can help readers think beyond the outcome of their choices, derive multiple meanings, and experience a story with an impactful and coherent plot. We infer that the reader's desire and the designer's goals do not have to be in conflict with each other as readers want to give themselves the best possible experience and act in rational ways.

In future studies, we will build a more robust and customizable installation with a dome and video projections over the wall, where readers can personalize ambient effects and how they map to moods. We will study how we can create diegetic interfaces that can be extended beyond Rangolis and Indian cultural context. We plan to study existing narrative-based media and interactive TV/film with the lens of agency to identify opportunities for meaningful interaction, more interaction styles, and suggestions for design. It is worth considering additional ways in which agency can be provided in interactive narratives. For example, hearing the story from different character perspectives [20], traversing non-linear narratives [41], and deepening narrative arcs $[6,21]$ are other possible ways that do not require plot changes. Researchers can study agency and apply these ideas in other domains such as smart homes. We hope this study can help designers identify the rich interpretive and reflective possibilities of decoupling the reader's interactions from the progress of the plot. We hope that our design lessons and levels of agency identified can guide researchers in creating interactive narratives.

\section{REFERENCES}

[1] Espen J. Aarseth. 1994. Nonlinearity and literary theory. Hyper/text/theory 52: 761-780.

[2] Ernest Adams. 1999. The Designer's Notebook: Three Problems for Interactive Storytellers. Gamasutra. Retrieved May 15, 2014 from 
http://www.gamasutra.com/view/feature/3414/the_des igners_notebook_three_.php

[3] Jim Bizzocchi. 2007. Games and Narrative: An Analytical Framework. Loading - The Journal of the Canadian Games Studies Association 1, 1: 5-10.

[4] Jim Bizzocchi. 2008. Ambient video: the transformation of the domestic cinematic experience. Small Tech: The Culture of Digital Tools 22: 197-206.

[5] Jim Bizzocchi, Ben Lin, and Theresa Jean Tanenbaum. 2011. Games, Narrative, and the Design of Interface. International Journal of Art and Technology (IJART) 4, 4: 460-479.

[6] Jim Bizzocchi and Theresa Jean Tanenbaum. 2012. Mass Effect 2: A Case Study in the Design of Game Narrative. Bulletin of Science Technology Society 32, 5: 393-404.

[7] David Bordwell and Kristin Thompson. 1997. Film Art: An Introduction. The McGraw Hill Companies, Inc., New York.

[8] Ronan Champagnat, Pascal Estraillier, and Armelle Prigent. 2006. Adaptative Execution of Game: Unfolding a Correct Story. In Proceedings of the 2006 ACM SIGCHI International Conference on Advances in Computer Entertainment Technology (ACE '06). https://doi.org/10.1145/1178823.1178941

[9] Juliet Corbin and Anselm Strauss. 2014. Basics of Qualitative Research. Sage Publications.

[10] Chris Crawford. 2017. Storytron: Interactive Storytelling. Retrieved September 13, 2018 from https://www.storytron.com/

[11] John W Creswell. 2003. Research Design: Qualitative, Quantitative, and Mixed Methods Approaches. Sage Publications, Thousand Oaks.

[12] Petra Fagerberg, Anna Ståhl, and Kristina Höök. 2004. eMoto: Emotionally Engaging Interaction. Personal Ubiquitous Comput. 8, 5: 377-381. https://doi.org/10.1007/s00779-004-0301-z

[13] Steve Gaynor. 2008. Being There. Being There (Fullbright). Retrieved May 15, 2014 from http://www.fullbrightdesign.com/2008/07/beingthere.html

[14] Saumya Gupta, Theresa Jean Tanenbaum, and Karen Tanenbaum. 2019. Shiva's Rangoli: Tangible Storytelling Through Diegetic Interfaces in Ambient Environments. In Proceedings of the Thirteenth International Conference on Tangible, Embedded, and Embodied Interaction (TEI '19), 65-75. https://doi.org/10.1145/3294109.3295635

[15] Daniel Harley, Jean Ho Chu, Jamie Kwan, and Ali Mazalek. 2016. Towards a Framework for Tangible Narratives. In Proceedings of the TEI '16: Tenth International Conference on Tangible, Embedded, and Embodied Interaction (TEI '16), 62-69. https://doi.org/10.1145/2839462.2839471

[16] Hiroshi Ishii and Brygg Ullmer. 1997. Tangible Bits: Towards Seamless Interfaces Between People, Bits and Atoms. In Proceedings of the ACM SIGCHI Conference on Human Factors in Computing Systems (CHI '97), 234-241. https://doi.org/10.1145/258549.258715

[17] Glena H. Iten, Sharon T. Steinemann, and Klaus Opwis. 2018. Choosing to Help Monsters: A MixedMethod Examination of Meaningful Choices in Narrative-Rich Games and Interactive Narratives. In Proceedings of the 2018 CHI Conference on Human Factors in Computing Systems (CHI '18), 341:1341:13. https://doi.org/10.1145/3173574.3173915

[18] Michelle Li and He Jianting. 2009. Ambient Environments for Emotional Physical Communication. In Proceedings of the 10th International Conference NZ Chapter of the ACM'S Special Interest Group on Human-Computer Interaction (CHINZ '09), 81-84. https://doi.org/10.1145/1577782.1577797

[19] Brian Magerko and John E. Laird. 2005. Mediating the tension between plot and interaction. Ann Arbor 1001: 48109-2110.

[20] Alexandra Mazalek. 2001. Tangible interfaces for interactive point-of-view narratives. Massachusetts Institute of Technology. Retrieved September 21, 2018 from http://dspace.mit.edu/handle/1721.1/61854

[21] Ali Mazalek, Glorianna Davenport, and Hiroshi Ishii. 2002. Tangible Viewpoints: A Physical Approach to Multimedia Stories. In ACM Conference on Multimedia. Retrieved from http://citeseerx.ist.psu.edu/viewdoc/download?doi=10 1.1.6.4303\&rep $=$ rep1\&type $=$ pdf

[22] Josh McCoy, Mike Treanor, Ben Samuel, Noah Wardrip-Fruin, and Michael Mateas. 2011. Comme Il Faut: A System for Authoring Playable Social Models. In Proceedings of the Seventh AAAI Conference on Artificial Intelligence and Interactive Digital Entertainment (AIIDE'11), 158-163. Retrieved from http://dl.acm.org/citation.cfm?id=3014589.3014617

[23] Janet Murray. 1997. Hamlet on the Holodeck: the future of narrative in cyberspace. The MIT Press, Cambridge, Massachusetts.

[24] Jeff L. Nay and José P. Zagal. 2017. Meaning Without Consequence: Virtue Ethics and Inconsequential Choices in Games. In Proceedings of the 12th International Conference on the Foundations of Digital Games (FDG '17), 14:1-14:8. https://doi.org/10.1145/3102071.3102073

[25] Mark Riedl. 2004. Narrative Generation: Balancing Plot and Character.

[26] Mark O. Riedl and R. Michael Young. 2006. From Linear Story Generation to Branching Story Graphs. IEEE Computer Graphics and Applications 26, 3: 2331.

[27] David Roberts, Charles L. Isbell Jr, Mark Riedl, Ian Bogost, and Merrick L. Furst. 2008. On the Use of Computational Models of Influence for Managing 
Interactive Virtual Experiences. Springer Berlin / Heidelberg, Berlin.

[28] Philip Ross and David V. Keyson. 2007. The Case of Sculpting Atmospheres: Towards Design Principles for Expressive Tangible Interaction in Control of Ambient Systems. Personal Ubiquitous Comput. 11, 2: 69-79. https://doi.org/10.1007/s00779-005-0062-3

[29] James A. Russell. 1980. A circumplex model of affect. Journal of Personality and Social Psychology 39, 6: 1161-1178. https://doi.org/10.1037/h0077714

[30] Katie Salen and Eric Zimmerman. 2003. Rules of Play: Game Design Fundamentals. MIT Press, Cambridge, MA; London, England.

[31] George J. Schafer, Susan King Fullerton, Ian Walker, Amith Vijaykumar, and Keith Evan Green. 2018. Words Become Worlds: The LIT ROOM, a Literacy Support Tool at Room-Scale. In Proceedings of the 2018 Designing Interactive Systems Conference (DIS '18), 511-522. https://doi.org/10.1145/3196709.3196728

[32] Magy Seif El-Nasr. 2004. An Interactive Narrative Architecture based on Filmmaking Theory. International Journal on Games and Simulation 3, 1: 29-36.

[33] Magy Seif El-Nasr. 2004. A User-centric Adaptive Story Architecture: Borrowing from Acting Theories. In Proceedings of the 2004 ACM SIGCHI International Conference on Advances in Computer Entertainment Technology (ACE '04), 109-116. https:// doi.org/10.1145/1067343.1067356

[34] Magy Seif El-Nasr. 2007. Interaction, Narrative, and Drama Creating an Adaptive Interactive Narrative using Performance Art Theories. Interaction Studies 8, 2.

[35] Anna Stla ahl, Petra Sundström, and Kristina Höök. 2005. A Foundation for Emotional Expressivity. In Proceedings of the 2005 Conference on Designing for User eXperience (DUX '05). Retrieved September 21, 2018 from http://dl.acm.org/citation.cfm?id=1138235.1138274

[36] Susan Leigh Star and James R. Griesemer. 1989. Institutional ecology,translations' and boundary objects: Amateurs and professionals in Berkeley's Museum of Vertebrate Zoology, 1907-39. Social studies of science 19, 3: 387-420.

[37] Nicolas Szilas. 2003. IDtension: A narrative engine for Interactive Drama. In Conference on Technologies for Interactive Digital Storytelling and Entertainment. Retrieved from https://www.academia.edu/21023655/IDtension_a_na rrative_engine_for_Interactive_Drama

[38] Theresa Jean Tanenbaum. 2011. Being in the Story: Readerly Pleasure, Acting Theory, and Performing a Role. In Interactive Storytelling, Mei Si, David Thue, Elisabeth André, James C. Lester, Theresa Jean Tanenbaum and Veronica Zammitto (eds.). Springer Berlin Heidelberg, 55-66. Retrieved May 22, 2014 from http://link.springer.com.proxy.lib.sfu.ca/chapter/10.10 07/978-3-642-25289-1_7

[39] Theresa Jean Tanenbaum. 2013. How I Learned to Stop Worrying and Love the Gamer: Reframing Subversive Play in Story-Based Games. In DiGRA 2013: DeFragging Game Studies. Retrieved from http://www.digra.org/wp-content/uploads/digitallibrary/paper_136.pdf

[40] Theresa Jean Tanenbaum. 2015. Identity Transformation and Agency in Digital Narratives and Story Based Games. Simon Fraser University, Surrey, British Columbia, Canada. Retrieved from http://summit.sfu.ca/item/15285

[41] Theresa Jean Tanenbaum, Karen Tanenbaum, and Alissa Antle. 2010. The Reading Glove: Designing Interactions for Object-based Tangible Storytelling. In Proceedings of the 1st Augmented Human

International Conference (AH '10), 19:1-19:9. https:// doi.org/10.1145/1785455.1785474

[42] Theresa Jean Tanenbaum and Angela Tomizu. 2007. Affective Interaction Design and Narrative Presentation. 150-157. Retrieved from http://www.aaai.org/Papers/Symposia/Fall/2007/FS7-05/FS07-05-025.pdf

[43] Theresa Jean Tanenbaum and Angela Tomizu. 2008. Narrative Meaning Creation in Interactive Storytelling. International Journal of Computational Science 2, 1: $3-20$.

[44] Karen Tanenbaum and Theresa Jean Tanenbaum. 2009. Commitment to Meaning: A Reframing of Agency in Games. In Digital Arts and Culture Conference, 2009: after media: embodiment and context. Retrieved from https://escholarship.org/uc/item/6f49r74n

[45] Karen Tanenbaum, Theresa Jean Tanenbaum, Alissa N. Antle, Jim Bizzocchi, Magy Seif el-Nasr, and Marek Hatala. 2011. Experiencing the Reading Glove. In Proceedings of the Fifth International Conference on Tangible, Embedded, and Embodied Interaction (TEI '11), 137-144. https://doi.org/10.1145/1935701.1935728

[46] David Thue, Vadam Bulitko, and Marcia Spetch. 2008. Making Stories Player-Specific: Delayed Authoring in Interactive Storytelling. In Interactive Storytelling, Ulrike Spierling and Nicolas Szilas (eds.). Springer Berlin / Heidelberg, Berlin, 230-241.

[47] K.C. Wassermann, Kynan Eng, P.F.M.J. Verschure, and J. Manzolli. 2003. Live soundscape composition based on synthetic emotions. IEEE Multimedia 10, 4: 82-90. https://doi.org/10.1109/MMUL.2003.1237553

[48] 2018. Ranjit Makkuni. Wikipedia. Retrieved from https://en.wikipedia.org/w/index.php?title=Ranjit_Ma kkuni\&oldid=823101557 